\title{
Feasibility results of an electromagnetic compatibility test protocol to evaluate medical devices to radio frequency identification exposure
}

\author{
Seth J Seidman ${ }^{\text {** }}$, Omar Bekdash², Joshua Guag², Maryam Mehryar ${ }^{3}$, Paul Booth $^{3}$ and Paul Frisch ${ }^{3}$
}

\author{
* Correspondence: \\ seth.seidman@fda.hhs.gov \\ ${ }^{1}$ FDA / CDRH / OSEL, 10903 New \\ Hampshire Ave, WO Bldg 62 Room \\ 1134, Silver Spring, MD 20993, USA \\ Full list of author information is \\ available at the end of the article
}

\begin{abstract}
Background: The use of radio frequency identification (RFID) systems in healthcare is increasing, and concerns for electromagnetic compatibility (EMC) pose one of the biggest obstacles for widespread adoption. Numerous studies have demonstrated that RFID systems can interfere with medical devices; however, the majority of past studies relied on time-consuming and burdensome test schemes based on ad hoc test methods applied to individual RFID systems.

Methods: This paper presents the results of using an RFID simulator that allows for faster evaluation of RFID-medical device EMC against a library of RFID test signals at various field strengths.
\end{abstract}

Results: The results of these tests demonstrate the feasibility and adequacy of simulator testing and can be used to support its incorporation into applicable consensus standards.

Conclusions: This work can aid the medical device community in better assessing the risks associated with medical device exposure to RFID.

Keywords: Electromagnetic compatibility, Electromagnetic interference, EMC, EMI, Radio-frequency identification, RFID, Medical device, Test methods, Simulator

\section{Background}

The use of radio frequency identification (RFID) systems in healthcare is increasing and concerns for electromagnetic compatibility (EMC) pose one of the biggest obstacles for widespread adoption. Numerous studies have demonstrated that RFID systems can interfere with medical devices [1-8] and previous ad hoc testing by FDA [8] demonstrated the need for a standardized test method, as it is impractical and time consuming to test for EMC between a medical device and individual RFID systems. RFID signal output, field strength, frequency, and separation distance are all factors that can contribute to the likelihood of electromagnetic interference (EMI). IEC 60601-1-2:2007 (the EMC test standard for non-implantable medical devices) has no immunity requirements at $125 \mathrm{kHz}$ nor a radiated immunity requirement at 13.56 $\mathrm{MHz}$. Additionally, the radiated immunity requirements at $915 \mathrm{MHz}$ and 2.4 $\mathrm{GHz}$ do not represent the potential field strengths allowed by the FCC. As such,

(c) 2014 Seidman et al.; licensee BioMed Central Ltd. This is an Open Access article distributed under the terms of the Creative Commons Attribution License (http://creativecommons.org/licenses/by/4.0), which permits unrestricted use, distribution, and reproduction in any medium, provided the original work is properly credited. The Creative Commons Public Domain Dedication waiver (http://creativecommons.org/publicdomain/zero/1.0/) applies to the data made available in this article, unless otherwise stated. 
medical devices tested to IEC 60601-1-2 can still be susceptible to RFID emissions as shown by Seidman et al. [1,8].

Because there are currently no standards that specify tests for medical device immunity to RFID emissions, medical device manufacturers typically demonstrate immunity through ad hoc testing, which has been shown to be both time consuming and labor intensive [8]. Previously it has been shown that by utilizing an RFID signal simulator it might be possible to test for a variety of RFID signals and field strengths, without the need for actual RFID systems [9]. Simulators described in detail in [10] are intended to provide an alternative means of testing for RFID emissions that is both faster and more reproducible. This type of testing would also provide medical device manufacturers with a better understanding of the RFID systems that could affect their devices, and this information can be used to improve device design.

The research described in this paper was performed to determine (1) simulator testing feasibility and adequacy and (2) if a broad range of input signals is necessary or if the test library can be simplified. Evaluation of the feasibility and adequacy of this simulator and the test library is important to support their incorporation into applicable medical device EMC standards.

\section{Methods}

As discussed in Seidman et al. [10], separate simulators were developed to cover four distinct RFID frequency bands: Low frequency (LF): $125 \mathrm{kHz}$; High frequency (HF): 13.56 MHz; Ultra high frequency (UHF): $915 \mathrm{MHz}$; and 2.4 GHz. At $125 \mathrm{kHz}$ and 13.56 MHz, Helmholtz Coils were designed to produce the radiated magnetic field strength (see Figure 1). At $915 \mathrm{MHz}$ and $2.4 \mathrm{GHz}$, the exposure setup was similar to that described by IEC 61000-4-3, with an adjusted input signal and field strength according to the RFID test library (see Figure 2). The input signals are intended to emulate the applicable RFID standards with the various RFID reader settings adjusted to create a signal with the maximum and minimum occupied bandwidth. A summary of these signals, named the RFID Test Library is presented in Table 1.

An infusion pump and three different patient monitors were tested against the RFID Test Library. To begin, each medical device was placed on a non-conductive surface and configured for normal operation. Normal operation for the infusion pump was specified as delivering saline at its minimum flow rate of $100 \mathrm{ml} / \mathrm{hr}$. Normal operation for

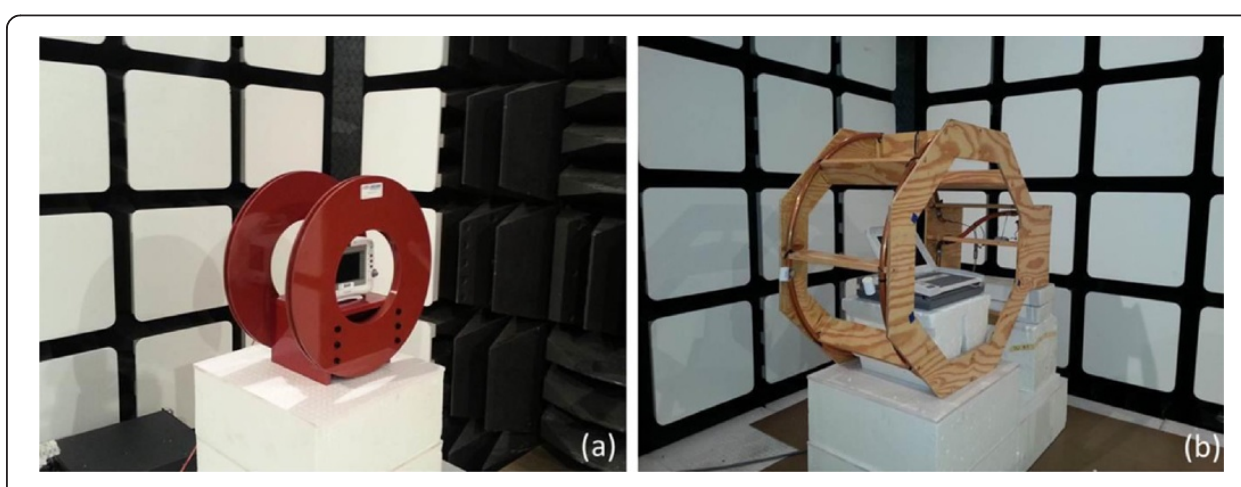

Figure 1 Magnetic field exposure systems. Photos of $125 \mathrm{kHz}$ (a) and $13.56 \mathrm{MHz}$ (b) exposure systems. 


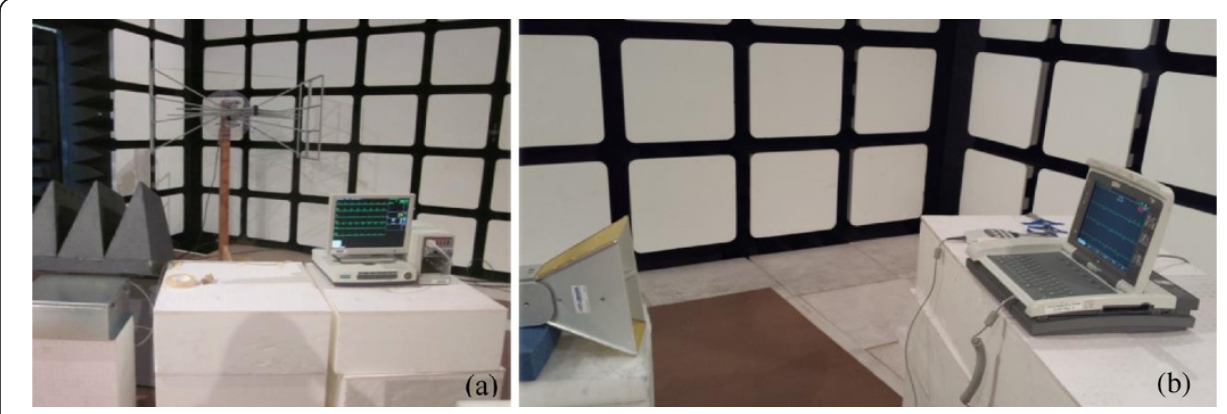

Figure 2 Electric field exposure systems. Photos of $915 \mathrm{MHz}$ (a) and $2.4 \mathrm{GHz}$ (b) exposure systems.

the patient monitors was correct detection and analysis of signals from a patient simulator (Dynatech Nevada Inc, Model 214A) set to output a normal sinus rhythm at 80 beats per minute to the acquisition module of the monitor. The patient simulator was placed in a shielded box to exclude any EMI that might have occurred with the simulator itself. We began testing the patient monitor with the acquisition module exposed as it would be configured in clinical use. However, the acquisition modules were extremely susceptible to EMI, and as the objective of this testing was to determine the feasibility of the test protocol and appropriateness of the RFID Test Library; we decided to gather additional data with the acquisition module shielded from exposure.

After normal operation of the device was confirmed, each device was exposed to an RFID test signal at maximum field strength from the RFID test library. Each device was exposed to four orientations for each RFID test signal: front, back, left, and right. Depending on the implementation and geometry of the device it could be helpful to expose the top and bottom orientation as well. Magnetic field testing in a Helmholtz coil is somewhat more efficient than electric field radiated testing in a chamber because the parallel (or perpendicular) orientation exposes both the front and back (or left and right) sides simultaneously. All medical devices were visually and audibly monitored during exposure with a surveillance video camera. Any observable changes in device operation were recorded for all tests and classified based on the severity of the interference. If EMI was observed at the maximum field strength, we performed an additional test to discover the threshold at which EMI began. During threshold testing, the field strength was increased in increments of approximately $10 \%$ from $1 \mathrm{~A} / \mathrm{m}$ to $12 \mathrm{~A} / \mathrm{m}$, until the first instance of EMI was observed. After EMI occurred, the field strength was lowered and the device was verified to return to normal operation. Next the field was returned to the level that caused the EMI and the threshold was verified. The dwell time for each exposure was 15 seconds.

\section{Results}

Observed EMI ranged from device mode changes to screen errors. Device mode changes and screen errors that made display information unreadable were considered probably clinically significant (Class I). Small display changes where the display information was still identifiable were considered probably not clinically significant (Class II).

We observed EMI (Class I or II) during 24\% (75 of 312) of our maximum field strength experiments with 76\% (57 of 75) being classified as Class I. EMI was observed during 25\% (6 of 24) of maximum field strength tests at $125 \mathrm{kHz}$; during 100\% (48 of 
Table 1 The RFID Test library

\begin{tabular}{|c|c|c|c|c|}
\hline Frequency & $\begin{array}{c}\text { Occupied } \\
\text { bandwidth }\end{array}$ & Standard & Program settings & $\begin{array}{l}\text { Maximum field } \\
\text { strength }\end{array}$ \\
\hline \multirow[t]{4}{*}{$125 \mathrm{kHz}$ (LF) } & Max & ISO 14223 Type A & Modulation Depth 100\% & $65 \mathrm{~A} / \mathrm{m}$ \\
\hline & & & ETU: 3 usec & \\
\hline & Min & ISO 14223 Type A & Modulation Depth $90 \%$ & $65 \mathrm{~A} / \mathrm{m}$ \\
\hline & & & ETU: $12 \mathrm{u} \mathrm{sec}$ & \\
\hline \multirow[t]{20}{*}{$13.56 \mathrm{MHz}(\mathrm{HF})$} & Max & ISO 14443 Type A & Pulse Width: 3 usec & $12 \mathrm{~A} / \mathrm{m}$ \\
\hline & & & Transition Edge: $1 \mathrm{nsec}$ & \\
\hline & Min & ISO 14443 Type A & Pulse Width: 2 usec & $12 \mathrm{~A} / \mathrm{m}$ \\
\hline & & & Transition Edge: 2.98 usec & \\
\hline & Max & ISO 14443 Type B & Modulation Depth: $25 \%$ & $12 \mathrm{~A} / \mathrm{m}$ \\
\hline & & & Transition Edge: 0.001 usec & \\
\hline & & & SOF Low: 11 etu & \\
\hline & & & SOF High: 2.5 etu & \\
\hline & & & EOF Low: 10.5 etu & \\
\hline & Min & ISO 14443 Type B & Modulation Depth: 15\% & $12 \mathrm{~A} / \mathrm{m}$ \\
\hline & & & Transition Edge: 1.17 usec & \\
\hline & & & SOF Low: 10 etu & \\
\hline & & & SOF High: 2 etu & \\
\hline & & & EOF Low: 10 etu & \\
\hline & Max & ISO 15693 & Modulation Depth: 100\% & $12 \mathrm{~A} / \mathrm{m}$ \\
\hline & & & Pulse Width: 9.44 usec & \\
\hline & & & Transition Edge: 1 nsec & \\
\hline & Min & ISO 15693 & Modulation Depth: 10\% & $12 \mathrm{~A} / \mathrm{m}$ \\
\hline & & & Pulse Width: 7.5 usec & \\
\hline & & & Transition Edge: 800 nsec & \\
\hline \multirow[t]{14}{*}{$915 \mathrm{MHz}$ (UHF) } & Max & ISO 18000-6C & Modulation: DSB-ASK & $54 \mathrm{~V} / \mathrm{m}$ \\
\hline & & & Tari: 6.25 us & \\
\hline & & & Pulse Width: 3.281 usec & \\
\hline & & & Modulation Depth: 100\% & \\
\hline & Max & ISO 18000-6C & Modulation: DSB-ASK & $54 \mathrm{~V} / \mathrm{m}$ \\
\hline & & & Tari: 25 us & \\
\hline & & & Pulse Width: 0.265 Tari & \\
\hline & & & Modulation Depth: $80 \%$ & \\
\hline & Min & ISO 18000-6C & Modulation: PR-ASK & $54 \mathrm{~V} / \mathrm{m}$ \\
\hline & & & Tari: 6.25 usec & \\
\hline & & & Pulse Width: 1.656 usec & \\
\hline & Min & ISO 18000-6C & Modulation: PR-ASK & $54 \mathrm{~V} / \mathrm{m}$ \\
\hline & & & Tari: 25 usec & \\
\hline & & & Pulse Width: 0.525 Tari & \\
\hline \multirow[t]{6}{*}{$2.45 \mathrm{GHz}$} & Max & ISO 18000-4 Mode 1 & Modulation Depth: 100\% & $54 \mathrm{~V} / \mathrm{m}$ \\
\hline & & & Transition Edge: 1 nsec & \\
\hline & & & Tari: 25 usec & \\
\hline & Min & ISO 18000-4 Mode 2 & Modulation Depth: 90\% & $54 \mathrm{~V} / \mathrm{m}$ \\
\hline & & & Transition Edge: 400 nsec & \\
\hline & & & Tari: 33 usec & \\
\hline
\end{tabular}

The RFID Test Library is a collection of test signals and field strengths that are representative for most commercially available RFID readers (RMS). 
48) of maximum field strength tests at $13.56 \mathrm{MHz}$; during 12\% (19 of 160) of maximum field strength tests at $915 \mathrm{MHz}$; and during 3\% (2 of 80) of maximum field strength tests at 2.4 GHz. The numbers above are inclusive of all tests, including experiments that were originally performed with the patient acquisition module exposed; for the remainder of this section we will ignore those experiments to minimize variables and concentrate purely on the appropriateness of the RFID Test Library.

During maximum field strength exposure to $13.56 \mathrm{MHz}$ test signals, we observed EMI in $100 \%$ (48 of 48 ) of the tests. Class I EMI was observed for all three patient monitors and Class II EMI was observed for the infusion pump. No EMI was observed during exposure to $125 \mathrm{kHz}$ (0 of 16), $915 \mathrm{MHz}$ (0 of 128) and 2.4 GHz (0 of 64) RFID test signals.

At the maximum field strength it was not possible to determine any effect of different RFID test signals within each frequency band because every $13.56 \mathrm{MHz}$ RFID test signal caused EMI. Thus for these cases where EMI was observed, we also found the field strength threshold where EMI began. We could then use these threshold values to compare the effects of different RFID tests signals within each frequency band. There were six 13.56 MHz RFID test signals (three different standards each tested at both maximum and minimum bandwidth). Results for these tests are presented in Table 2 below:

Table 2 Threshold EMI values at $13.56 \mathrm{MHz}$ for parallel and perpendicular orientations for all devices

\begin{tabular}{|c|c|c|c|}
\hline \multirow[t]{2}{*}{ Device \# } & \multirow{2}{*}{$\begin{array}{l}\text { RFID Standard } \\
\text { protocol }\end{array}$} & \multicolumn{2}{|c|}{ Threshold H-Field strength (A/m RMS) } \\
\hline & & Parallel & Perpendicular \\
\hline \multirow[t]{6}{*}{1} & ISO 14443 Type A Max & 4.5 & 4.5 \\
\hline & ISO 14443 Type A Min & 4.5 & 6.5 \\
\hline & ISO 14443 Type B Max & 4.5 & 4 \\
\hline & ISO 14443 Type B Min & 4 & 4 \\
\hline & ISO 15693 Max & 4 & 3.5 \\
\hline & ISO 15693 Min & 4.5 & 3.5 \\
\hline \multirow[t]{6}{*}{2} & ISO 14443 Type A Max & 10.5 & 12 \\
\hline & ISO 14443 Type A Min & 10.5 & 12 \\
\hline & ISO 14443 Type B Max & 10.5 & 9.5 \\
\hline & ISO 14443 Type B Min & 10.5 & 12 \\
\hline & ISO 15693 Max & 10.5 & 11.5 \\
\hline & ISO 15693 Min & 10.5 & 11.5 \\
\hline \multirow[t]{6}{*}{3} & ISO 14443 Type A Max & 12 & 12 \\
\hline & ISO 14443 Type A Min & 12 & 12 \\
\hline & ISO 14443 Type B Max & 12 & 12 \\
\hline & ISO 14443 Type B Min & 12 & 12 \\
\hline & ISO 15693 Max & 12 & 12 \\
\hline & ISO 15693 Min & 12 & 12 \\
\hline \multirow[t]{6}{*}{4} & ISO 14443 Type A Max & 12 & 12 \\
\hline & ISO 14443 Type A Min & 12 & 12 \\
\hline & ISO 14443 Type B Max & 12 & 12 \\
\hline & ISO 14443 Type B Min & 12 & 12 \\
\hline & ISO 15693 Max & 12 & 12 \\
\hline & ISO 15693 Min & 12 & 12 \\
\hline
\end{tabular}




\section{Discussion}

This study aimed to determine (1) simulator testing feasibility and adequacy and (2) if all proposed input signals are necessary or if the test library can be simplified. Test feasibility and adequacy was verified by demonstrating that the simulator can be used to determine medical device-RFID EMC. Little variation was seen comparing the different RFID protocols and the maximum versus minimum bandwidth. The average difference comparing threshold field strengths between different standards was only $0.25 \mathrm{~A} / \mathrm{m}$ and the average difference comparing the maximum versus the minimum bandwidth was only $0.23 \mathrm{~A} / \mathrm{m}$. Additionally, identical EMI was observed for all patient monitors tested across the six protocols. While only four medical devices were tested, this data collected tends to suggest that, while signal modulation has an effect, the greater influence is the overall field strength. This would support minimizing the RFID Test Library to one signal per frequency. However, threshold testing was only performed at $13.56 \mathrm{MHz}$ and more testing with more medical devices is needed to verify simplification of the RFID Test Library. Since little variation was seen between the different RFID protocols future tests should consider whether the specified modulation defined in IEC 60601-1-2:2007 is adequate with the appropriate higher test levels.

Varying EMC performance was seen between devices. Device 1 exhibited EMI at an average field strength of $4.3 \mathrm{~A} / \mathrm{m}$, while all of the other medical devices exhibited EMI at an average field strength between $11 \mathrm{~A} / \mathrm{m}$ and $12 \mathrm{~A} / \mathrm{m}$. While our results confirm the potential of RFID EMI to medical devices, we do not recommend using this data for clinical decisions. First, there were only four devices tested in this study and they do not represent a broad enough selection of medical device types or manufacturers. Secondly, the devices were overexposed for a portion of the testing performed. A maximum test level of $12 \mathrm{~A} / \mathrm{m}$ was chosen at $13.56 \mathrm{MHz}$ for all three different standards. However, while ISO 18000-3 Mode 1 specifies a maximum field strength of $12 \mathrm{~A} / \mathrm{m}$, ISO/IEC 14443 and ISO/IEC 15693 specify $7.5 \mathrm{~A} / \mathrm{m}$ and $5 \mathrm{~A} / \mathrm{m}$, respectively (over the manufacturer's specified volume). Similarly the $54 \mathrm{~V} / \mathrm{m}$ test level at $2.4 \mathrm{GHz}$ is a theoretical maximum at $20 \mathrm{~cm}$. In our experience the field values from $2.4 \mathrm{GHz}$ readers are less than $2 \mathrm{~V} / \mathrm{m}$ at this distance. Finally, during threshold testing the patient monitor acquisition modules were shielded from interference. Many medical devices sense vital signs from the body and a modulated RF signal can be misinterpreted as one coming from a patient by that unit if it is exposed. In clinical use, the acquisition module would be exposed, resulting in EMI at lower field strengths than those reported here.

A side by side comparison of our simulator data to actual RFID readers would be helpful to demonstrate that our test accurately portrays exposure from actual RFID readers. However, there are some practical reasons why these tests were not performed. Primarily this was not performed because testing to a maximum field in a volume or over an area as described in our test method is generally understood to be a more rigorous exposure than testing with an actual RFID reader, where the maximum field will be localized and decrease with distance. This means that passing our test is more difficult than passing a test with actual RFID readers. Additionally, we were not able to reproduce all of the field levels with actual RFID readers, as explained in the prior paragraph. We did do some testing using actual RFID readers as the signal input, but with our same exposure system. As expected, this data was consistent with that found from using the signals from the RFID Test Library. 


\section{Conclusions}

Medical devices have been shown to be susceptible to EMI from RFID and current medical device EMC standards do not adequately test electromagnetic immunity of medical devices to RFID systems. This study has demonstrated the feasibility and adequacy of using simulators and the RFID Test Library to test medical devices for EMC with RFID. Using simulators, we have shown that testing can be performed much faster than in ad hoc testing with individual RFID systems. The results from our tests demonstrate that, while signal modulation has an effect on medical devices, the greater influence is the overall field strength. Tests are also more repeatable than in ad hoc testing because the hardware configuration remains unchanged from test to test and results are not affected by small changes in antenna orientation.

Medical device manufacturers could use these simulators to test if their devices are susceptible to RFID emissions. This test can be used to assist the medical device community to identify and resolve potential EMI that could be caused by RFID systems and provide assurance to hospitals that RFID technology will not adversely affect their medical devices. It has also been shown to be suitable for incorporation into applicable consensus standards.

\section{Abbreviations}

RFID: Radio frequency identification; EMC: Electromagnetic compatibility; EMI: Electromagnetic interference; LF: Low frequency; HF: High frequency; UHF: Ultra high frequency.

\section{Competing interests}

The authors declare that they have no competing interests.

\section{Authors' contributions}

All authors have made substantial contributions to the conception and design, acquisition, analysis and interpretation of data. All authors were involved in drafting the manuscript. All authors read and approved the final manuscript.

\section{Acknowledgement}

The authors would like to acknowledge the contributions of Paul Ruggera who contributed to the acquisition of data.

\section{Disclaimer}

The mention of commercial products, their sources, or their use in connection with material reported herein is not to be construed as either an actual or implied endorsement of such products by the Department of Health and Human Services.

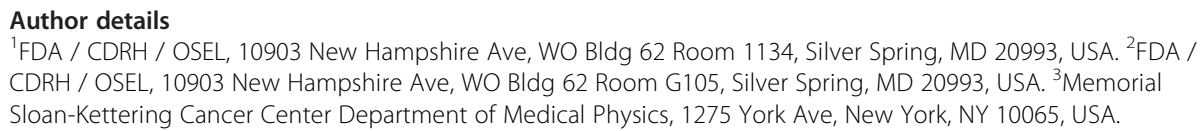

Received: 16 May 2014 Accepted: 25 July 2014

Published: 3 August 2014

\section{References}

1. Seidman SJ, Brockman R, Lewis BM, Guag J, Shein MJ, Clement WJ, Kippola J, Digby D, Barber C, Huntwork D: In vitro tests reveal sample radiofrequency identification readers inducing clinically significant electromagnetic interference to implantable pacemakers and implantable cardioverter-defibrillators. Heart Rhythm 2010, 7:99-107.

2. Pantchenko OS, Seidman SJ, Guag JW, Witters DM Jr, Sponberg CL: Electromagnetic compatibility of implantable neurostimulators to RFID emitters. Biomed Eng Online 2011, 10:50.

3. van der Togt R, van Lieshout EJ, Hensbroek R, Beinat E, Binnekade JM, Bakker PJM: Electromagnetic interference from radio frequency identification inducing potentially hazardous incidents in critical care medical equipment. JAMA 2008, 299:2884-2890.

4. Futatsumori S, Hikage T, Nojima T, Koike B, Fujimoto H, Toyoshima T: A Novel Assessment Methodology for the EMI Occurrence in Implantable Medical Devices Based Upon Magnetic Flux Distribution of RFID Reader/ Writers. In Electromagnetic Compatibility, 2007 EMC 2007 IEEE International Symposium on; 9-13 July 2007. New York, NY: IEEE; 2007:1-6.

5. Ogirala A, Stachel JR, Mickle MH: Electromagnetic interference of cardiac rhythmic monitoring devices to radio frequency identification: analytical analysis and mitigation methodology. IEEE Trans Inf Technol Biomed 2011, 15:848-853. 
6. Houliston B, Parry D, Webster CS, Merry AF: Interference with the operation of medical devices resulting from the use of radio frequency identification technology. N Z Med J 2009, 122:9-16.

7. Ying Y: Electromagnetic Interference With RFID Readers in Hospitals. In IFMBE. Berlin, Germany: Springer; 2009:872-875.

8. Seidman S, Guag J: Adhoc electromagnetic compatibility testing of non-implantable medical devices and radio frequency identification. Biomed Eng Online 2013, 12(1):71.

9. Ogirala A, Stachel JR, Mickle MH: In vitro test modeling for electromagnetic compatibility evaluation between CRMDs and RFID systems. International Journal of Modelling and Simulation 2013, 33(2):86-92.

10. Seidman S, Pantchenko O, Tennakoon D: Design of Unique Simulators to Evaluate Medical Device Susceptibility to Radio Frequency Identification Exposure. In Electromagnetic Compatibility Magazine, Volume 3. New York, NY: IEEE; 2014:70-74.

doi:10.1186/1475-925X-13-110

Cite this article as: Seidman et al.: Feasibility results of an electromagnetic compatibility test protocol to evaluate medical devices to radio frequency identification exposure. BioMedical Engineering OnLine 2014 13:110.

\section{Submit your next manuscript to BioMed Central and take full advantage of:}

- Convenient online submission

- Thorough peer review

- No space constraints or color figure charges

- Immediate publication on acceptance

- Inclusion in PubMed, CAS, Scopus and Google Scholar

- Research which is freely available for redistribution

Submit your manuscript at www.biomedcentral.com/submit 